(2) LSM $>11 \mathrm{KPa}$ could be used to identify patients who may have lower response rates and may benefit from longer treatment.

\section{P19 \\ SYMPTOMATIC EPSTEIN BARR VIRUS (EBV) HEPATITIS IS UNCOMMON, BUT OCCURS IN PATIENTS OF ANY AGE, INCLUDING THE ELDERLY}

doi:10.1136/gutjnl-2011-300857a.19

L Vine, K Shephard, D Grimes, R Bendall, H Dalton. Royal Cornwall Hospital Trust

Introduction Epstein Barr Virus (EBV) infection typically presents as infectious mononucleosis (IM) in young adults with the classical triad of fever, sore throat and lymphadenopathy. Mildly abnormal liver function tests (LFTs) are common but symptomatic hepatitis is rare. Symptomatic, icteric, EBV hepatitis is rarely reported in those with IM and in the elderly.

Aim To review the demographics, presenting features and natural history with EBV hepatitis and to determine what factors might lead a clinician to consider a diagnosis of EBV hepatitis.

Method Retrospective study of 2100 patients attending the Jaundice Hotline, a fast track referral system for patients with jaundice at The Cornwall Royal Cornwall Hospital (1998-2011). EBV hepatitis was defined as: symptomatic hepatitis with positive serology for EBV (serology included EBV nuclear antigen, EBV viral capsid antigen (VCA) IgM and VCA IgG). All patients had no evidence of biliary obstruction or parenchymal liver disease on abdominal ultrasound examination (USS), and negative serology for other hepatotropic viruses (HAV, HBV, HCV, HEV, CMV). Other causes of parenchymal liver disease were also excluded by appropriate blood tests.

Results Of the total of 2100 consecutive patients with jaundice studied, 17 patients (10 males, 7 females) were diagnosed with EBV hepatitis. All patients were immunocompetent. 47\% (8/17) of these patients were aged over 60 years (mean age 44 years, range 18-82). At presentation, mean (range) LFTs were: bilirubin $57 \mu \mathrm{mol} / 1$ (11-161), ALT 428 IU/1 (34-1471), ALKP 339 IU/1 (132-840). Only 3 patients presented as classical IM. 95\% (16/17) had significant lymphocytosis and $82 \%(14 / 17)$ patients had splenomegaly on USS examination at initial assessment. Only 2 patients were unwell enough to be admitted to hospital. All patients fully recovered within 4-6 weeks.

Conclusion Symptomatic EBV hepatitis is uncommon and causes a self-limiting illness. EBV hepatitis is not usually associated with classical symptoms of IM and occurred in patients of a wide variety of ages. The diagnosis should be considered in patients of all ages presenting with hepatitis, including those with a cholestatic picture, and especially in those with a lymphocytosis and splenomegaly.

\section{P2O FIRST REPORT OF THE LONG-TERM EFFICACY OF A NOVEL ENDOSCOPIC RADIOFREQUENCY ABLATION TECHNIOUE FOR MALIGNANT BILIARY OBSTRUCTION}

doi:10.1136/gutjnl-2011-300857a.20

${ }^{1} \mathrm{Y}$ Kallis, ${ }^{1} \mathrm{~N}$ Phillips, ${ }^{2} \mathrm{~A}$ Steel, ${ }^{3} \mathrm{C}$ Baldwin, ${ }^{4} \mathrm{~J}$ Nicholls, ${ }^{4} \mathrm{~L}$ Jiao, ${ }^{1} \mathrm{P}$ Vlavianos, ${ }^{4} \mathrm{~N}$ Habib, ${ }^{1} \mathrm{D}$ Westaby. ${ }^{1}$ Department of Gastroenterology, Imperial College Healthcare, London; ${ }^{2}$ Department of Gastroenterology, Chelsea and Westminster Hospital, London; ${ }^{3}$ Faculty of Medicine, Imperial College, London; ${ }^{4}$ Academic Department of Surgery, Hammersmith Hospital, Imperial College, London

Introduction Insertion of self-expanding metal stents (SEMS) is standard practice in patients with unresectable malignant biliary strictures. Stent occlusion is a significant clinical problem in patients surviving beyond 3 months. A pioneering phase I/II study in our tertiary referral centre demonstrated good safety and 30-day patency using a novel endoscopic radiofrequency ablation (RFA) technique as

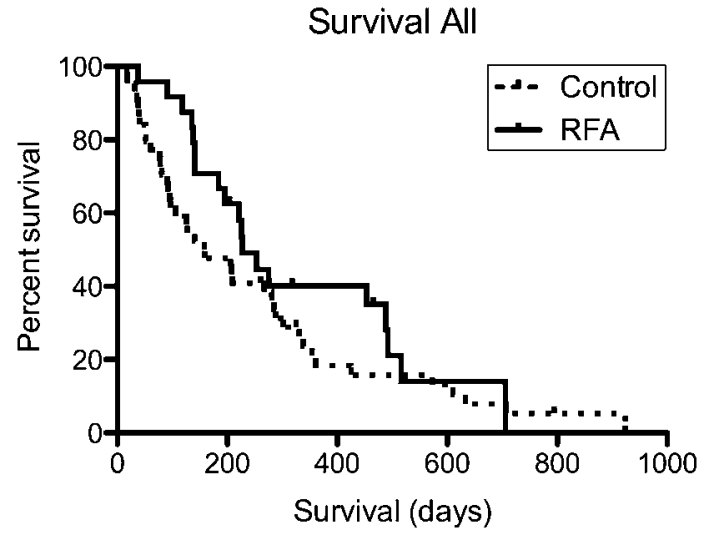

Abstract P20 Figure 1 Kaplan-Meier survival analysis.

an adjunct to SEMS. ${ }^{1}$ The longer term impact of combined RFA + SEMS on biliary drainage and overall patient survival is unknown. Aim To investigate long-term safety and efficacy of endobiliary RFA in malignant bile duct obstruction.

Method Retrospective cohort analysis of 24 patients undergoing RFA+SEMS (17 pancreatic carcinoma; 7 cholangiocarcinoma) and 44 matched controls undergoing SEMS insertion alone (34 pancreatic carcinoma, 10 cholangiocarcinoma) for malignant biliary obstruction in a single tertiary referral centre. Patients were matched for age, sex, disease, presence of metastases, ASA/co-morbidities, and intention to treat with palliative chemotherapy. Patients with a potential minimum of 6-month follow-up were included and survival, maintenance of stent patency and procedure-related complications were assessed.

Results RFA treated and control cohorts were closely matchedmean age $71.8 \pm 9.8$ yrs vs $68.8 \pm 10.3$, metastases at treatment $9 / 24$ (38\%) vs $17 / 44$ (39\%), chemotherapy $16 / 24$ (67\%) vs $27 / 44(61 \%)$. Kaplan-Meier analysis showed a median survival of 227 days in the RFA group vs 159 days in controls ( $p=0.067$ ). Multivariate analysis showed RFA treatment to be the strongest predictor of survival at 90 days (OR 26.1, $\mathrm{p}=0.011$ ). Survival benefits may extend beyond 90 days (OR 2.8, $\mathrm{p}=0.071$ at 180 days; $\mathrm{OR} 2.8, \mathrm{p}=0.102$ at 360 days), but require further investigation. Within 6 months after treatment, more patients were alive with a patent first SEMS in the RFA cohort than in controls. Complications of RFA were few (1 pancreatitis, 2 cholecystitis) and comparable to those associated with standard ERCP alone. The procedure was well-tolerated with a median post-procedure inpatient stay of 1 day (1-24).

Conclusion In the single largest case series studied to date, endobiliary RFA is a safe and efficacious treatment for malignant biliary obstruction, with potential early survival benefit. Large multi-centre prospective trials of this novel treatment modality are warranted.

\section{REFERENCE}

1. Steel AW, et al. Gastrointest Endosc 2011;73:149-53.

\section{P21 ACTIVELY INJECTING DRUG USERS CAN BE SUCCESSFULLY TREATED WITH ANTIVIRAL THERAPY FOR HCV, ARE UNLIKELY TO BE RE-INFECTED, AND SIGNIFICANTLY REDUCE THEIR ILLICIT DRUG USE}

doi:10.1136/gutjnl-2011-300857a.21

${ }^{1} \mathrm{H}$ Lewis, ${ }^{2} \mathrm{~N}$ Ibrahim, ${ }^{2} \mathrm{M}$ Mirza, ${ }^{2} \mathrm{~J}$ Hothi, ${ }^{3} \mathrm{M}$ Wilkinson, ${ }^{1} \mathrm{G}$ Foster. ${ }^{1}$ Queen Mary University of London; ${ }^{2}$ Barts and the London School of Medicine and Dentistry; ${ }^{3}$ East London NHS Foundation Trust

Introduction Currently the predominant mode of transmission of hepatitis $\mathrm{C}$ virus (HCV) in the developed world is injection drug use 
(IDU) and HCV antibody prevalence rates reach $60-90 \%$ in those who have injected drugs. Treatment rates of injecting drug users (IDU's) with HCV are low at just $3-10 \%$ and although small studies indicate treatment of IDU's is safe and effective, data on outcomes of antiviral therapy in current injecting drug users is limited with the largest study published to date including just 31 active IDU's. It has been suggested that antiviral therapy for HCV use may reduce illicit drug use, but published data to support this is lacking

Aim To assess the treatment outcomes and the impact of antiviral therapy on illicit drug use in injecting drug users (IDU's) treated with antiviral therapy for Hepatitis $\mathrm{C}$ virus (HCV) by nurses based in community addiction services in North East London. The outcomes of the first 81 patients to be treated are presented here, 48 of whom were using illicit drugs at the start of treatment making this the largest cohort of active IDU's with HCV treated with antiviral therapy that has been presented to date.

Method Methods consisted of a retrospective database analysis. Illicit drug use was quantified as 3 (heavy/daily); 2 (weekly/ moderate); 1 (monthly/occasional) and 0 (previous drug use). The Wilcoxon signed rank test was used for statistical analysis.

Results 81 patients were treated over a 45 -month period from September 2004 to January 2009. 50 (62\%) were genotype 2/3, and $31(38 \%)$ were genotype 1 . The average age of infection with HCV was 23 , of diagnosis was 39 and of treatment was 41 . Compliance with treatment was $88 \%$. $63 \%$ of patients had a sustained viral response, $18 \%$ were non-responders and $10 \%$ discontinued treatment early due to side effects. 5 patients $(6 \%)$ relapsed after successful treatment. Only 1 patient (1\%) has been re-infected post treatment. 48 patients $(60 \%)$ were actively using illicit drugs when HCV treatment was initiated. Data on pre and post treatment intravenous heroin use, crack cocaine use and alcohol use was available in 74, 40 and 21 patients respectively. Intravenous heroin use reduced from $41 / 74$ patients $(55 \%)$ to $27 / 74(36 \%)(p=0.0033)$ after treatment, with daily use reducing from $16 / 74(22 \%)$ to $7 / 74$ (9\%) Crack cocaine use reduced from $32 / 40(80 \%)$ to $21 / 40(53 \%)$ $(p=0.0103)$ Alcohol use reduced from $15 / 21(71 \%)$ to $13 / 21(62 \%)$ $(\mathrm{p}=0.5775)$.

Conclusion Nurse led provision of antiviral therapy for HCV infected injecting drug users in community based clinics is effective, with sustained viral response (SVR) rates that compare favourably with published randomised controlled trials of pegylated interferon and ribavirin. ${ }^{1}$ High rates of compliance are seen and re-infection rates are low, so concern over compliance and re-infection should not prevent treatment of injecting drug users. This study has shown for the first time that a significant reduction in illicit drug use occurs during and after antiviral therapy demonstrating a social benefit of treatment in addition to the known health benefits.

\section{REFERENCE}

1. Fried MW, Shiffman ML, Reddy KR. Peginterferon alfa-2a plus ribavirin for chronic Hepatitis C virus infection. N Engl J Med 2002:347.

\section{P22 \\ GALL BLADDER LENGTH ON ULTRSONOGRAPHY AS A SCREENING TOOL IN IDENTIFICATION OF AUTO IMMUNE SCLEROSING CHOLANGITIS}

doi:10.1136/gutjnl-2011-300857a.22

${ }^{1} \mathrm{P}$ McKiernan, ${ }^{1} \mathrm{~A}$ Batra, ${ }^{2} \mathrm{~K}$ Au-Yong, ${ }^{1} \mathrm{D}$ A Kelly, ${ }^{2} \mathrm{H}$ Alton. ${ }^{1}$ Liver Unit, Birmingham Children's Hospital; ${ }^{2}$ Radiology Department, Birmingham Children's Hospital

Introduction Autoimmune liver disease (AILD) in children encompasses Autoimmune Hepatitis (AIH), Autoimmune sclerosing Cholangitis (ASC) or, where there are features of both, overlap syndrome (OS). Differentiating between these entities requires cholangiography. The gold standard for the diagnosis of ASC is Endoscopic Retrograde Cholangiopancreaticography which is invasive. Magnetic Resonance Cholangiopancreaticography can be used as an alternative but is not universally available and may require anaesthesia in young children. Anecdotally, we had found increased gall bladder (GB) length on ultrasound to be a useful marker of bile duct involvement in AILD.

Aim Our aim was to study the role of ultrasound measurement of Gall Bladder length as a screening tool for identifying ASC in children with AILD.

Method Children, under the age of 18 years at the time of presentation, diagnosed with AILD were identified from the departmental database. Cases for whom a fasting ultrasound at the time of presentation, was available on our radiology department's digital imaging system were included. Cases were categorised using established criterion into 2 groups; those with AIH and those with ASC with or without OS. A retrospective case notes review was performed on all eligible children, their ultrasound scans were reviewed by a single radiologist and GB length measured.

Results 50 cases were included. 32/50 (64\%) had AIH type1 and 6/ 50 (12\%) had AIH type2. 12/50 (24\%) had ASC and 6/12 (50\%) of these had OS. The average age at presentation was 10.18 years (range 1.3-16 years). The age at presentation was similar for both groups (AIH 10.1 yrs; ASC 10.2 yrs). Overall there were 22 males and 28 females but among the group with ASC there were 8 males and 4 females. The duration of symptoms before diagnosis was 5 months and was similar in both groups. The median (25th, 75th centile) GB length in children with ASC was $9.75 \mathrm{~cm}(7.2 \mathrm{~cm}$, $10.6 \mathrm{~cm})$ and in children with AIH was $6.8 \mathrm{~cm}(5.5 \mathrm{~cm}, 7.9 \mathrm{~cm})$ [ $p$ value 0.003 ]. The normal GB length in children older than 1 year is up to $7 \mathrm{~cm}$. GB length $>7 \mathrm{~cm}$ was seen in $9 / 12(75 \%)$ children with ASC compared to 15/38 (39.5\%) of children with AIH $(p=0.04)$. GB length $>9 \mathrm{~cm}$ was seen in $7 / 12(58.3 \%)$ children with ASC and $4 / 38(10.5 \%)$ children with AIH ( $p=0.005)$.

\begin{tabular}{lllll}
\hline GB length & $\begin{array}{l}\text { Sensitivity } \\
(\%)\end{array}$ & $\begin{array}{l}\text { Specificity } \\
(\%)\end{array}$ & $\begin{array}{l}\text { +ve predictive } \\
\text { value }(\%)\end{array}$ & $\begin{array}{l}\text {-ve predictive } \\
\text { value }(\%)\end{array}$ \\
\hline$>7 \mathrm{~cm}$ & 75 & 59 & 38 & 88 \\
$<7 \mathrm{~cm}$ & 58 & 90 & 63 & 87 \\
\hline
\end{tabular}

Conclusion Summary: In AILD GB length is significantly increased in children with ASC. GB length $>9 \mathrm{~cm}$ has $90 \%$ specificity for the diagnosis of ASC. GB length $<7 \mathrm{~cm}$ is $88 \%$ exclusive of ASC.

Conclusion: GB length measurement is a useful screening test for ASC in children with AILD. We would recommend a further study with greater number of patients.

\section{P23 COMPARISON OF ENHANCED LIVER FIBROSIS TEST AND TRANSIENT ELASTOGRAPHY FOR THE NON-INVASIVE ASSESSMENT OF LIVER FIBROSIS IN CHRONIC HEPATITIS B}

doi:10.1136/gutjnl-2011-300857a.23

${ }^{1} \mathrm{P}$ M Trembling, ${ }^{2} \mathrm{P}$ Lampertico, ${ }^{3} \mathrm{~J}$ Parkes, ${ }^{1} \mathrm{~S}$ Tanwar, ${ }^{4} \mathrm{M}$ Viganú, ${ }^{2} \mathrm{~F}$ Facchetti, ${ }^{2} \mathrm{M}$ Colombo, ${ }^{1} \mathrm{~W}$ M Rosenberg. ${ }^{1}$ Centre for Hepatology, University College London, London, UK; ${ }^{2} 1$ st Division of Gastroenterology, Fondazione IRCCS Ca? Granda Ospedale Maggiore Policlinico, Universiț di Milano, Milan, Italy; ${ }^{3}$ Faculty of Medicine, University of Southampton, Southampton, UK; ${ }^{4}$ UO Epatologia, Ospedale San Giuseppe, Universitt degli Studi di Milano, Milan, Italy

Introduction The enhanced liver fibrosis (ELF) test comprises a panel of biomarkers of liver fibrosis shown to accurately assess fibrosis and 